\title{
Mother-to-Child Transmission of HIV Infection in the Era of Highly Active Antiretroviral Therapy
}

\author{
European Collaborative Study
}

\section{(See the editorial commentary by Luzuriaga and Sullivan on pages 466-7)}

Background. Very low rates of mother-to-child transmission (MTCT) of human immunodeficiency virus (HIV) are achievable with use of highly active antiretroviral therapy (HAART). We examine risk factors for MTCT in the HAART era and describe infants who were vertically infected, despite exposure to prophylactic MTCT interventions.

Methods. Of the 4525 mother-child pairs in this prospective cohort study, 1983 were enrolled during the period of January 1997 through May 2004. Factors examined included use of antiretroviral therapy during pregnancy, maternal CD4 cell count and HIV RNA level, mode of delivery, and gestational age in logistic regression analysis.

Results. Receipt of antenatal antiretroviral therapy increased from 5\% at the start of the HAART era to 92\% in 2001-2003. The overall MTCT rate in this period was 2.87\% (95\% confidence interval [CI], $2.11 \%-3.81 \%$ ), but it was $0.99 \%$ (95\% CI, $0.32 \%-2.30 \%)$ during 2001-2003. In logistic regression analysis that included 885 mother-child pairs, MTCT risk was associated with high maternal viral load (adjusted odds ratio [AOR], 12.1; $P=.003$ ) and elective Caesarean section (AOR, $0.33 ; P=.04$ ). Detection of maternal HIV RNA was significantly associated with antenatal use of antiretroviral therapy, CD4 cell count, and mode of delivery. Among 560 women with undetectable HIV RNA levels, elective Caesarean section was associated with a 90\% reduction in MTCT risk (odds ratio, 0.10; 95\% CI, 0.03-0.33), compared with vaginal delivery or emergency Caesarean section.

Conclusions. Our results suggest that offering an elective Caesarean section delivery to all HIV-infected women, even in areas where HAART is available, is appropriate clinical management, especially for persons with detectable viral loads. Our results also suggest that previously identified risk factors remain important.

In developed countries, the success of preventive interventions for mother-to-child transmission of HIV has resulted in transmission rates of $<2 \%[1-4]$ and the small numbers of vertically acquired pediatric infections now being reported $[5,6]$. Key to these dramatic decreases in mother-to-child transmission rates has been the availability of HAART, which is now used by most pregnant HIV-infected women in resource-rich

Received 9 July 2004; accepted 24 September 2004; electronically published 7 January 2005.

The views expressed are those of the authors and not necessarily those of the Medical Research Council or the United Kingdom health departments.

a This article was prepared by Claire Thorne, Deven Patel, Simona Fiore, Catherine Peckham, and Marie-Louise Newell (Centre for Paediatric Epidemiology and Biostatistics, Institute of Child Health, University College, London, United Kingdom). Members of the European Collaborative Study are listed at the end of the text.

Reprints or correspondence: Dr. Claire Thorne, Centre for Paediatric Epidemiology and Biostatistics, Institute of Child Health, 30 Guilford St., London, WC1N 1EH, UK (c.thorne@ich.ucl.ac.uk).

Clinical Infectious Diseases 2005; 40:458-65

(C) 2005 by the Infectious Diseases Society of America. All rights reserved. $1058-4838 / 2005 / 4003-0018 \$ 15.00$ countries, an increasing number of whom achieve undetectable viral loads before delivery [7]. The substantial effectiveness of elective Caesarean section delivery in reducing mother-to-child transmission of HIV was recognized in the monotherapy era [8-10], but with the success of HAART in reducing maternal viral load, the added benefit of this intervention has been questioned [11-13]. Whether the benefit of elective Caesarean section delivery occurs in women who receive HAART with low or undetectable viral loads is difficult to assess, given their low risk of mother-to-child transmission of HIV. Similarly, HAART use has been associated with premature birth [14], but it is unclear whether these premature infants are at higher risk of mother-to-child transmission of HIV than term infants, as has been reported elsewhere $[15,16]$.

Despite the range of interventions available and the usually timely identification of HIV-infected women during pregnancy, a small number of infants born to HIV-infected women continue to acquire infection in utero or during delivery. In this article, we examine 
risk factors for mother-to-child transmission of HIV in the European Collaborative Study during the HAART era. In particular, we investigate the effects of elective Caesarean section delivery, duration of rupture of membranes, and prematurity. We also describe the small number of infants who acquired HIV infection, despite maternal exposure to prophylactic interventions.

\section{METHODS}

The European Collaborative Study is an ongoing cohort study in which HIV-infected pregnant women were enrolled and their infants prospectively observed in accordance with standard clinical and laboratory protocols $[17,18]$. The European Collaborative Study was set up in 1985 and includes 29 centers in 10 European countries. Informed consent was obtained before enrollment, in accordance with local guidelines, and local ethics committee approval was granted. Information collected included timing of initiation and type of antiretroviral treatment, maternal CD4 cell count and viral load, history of injection drug use, and other sociodemographic characteristics. Information on maternal CD4 cell count was routinely collected in most study centers from 1992 onwards, and routine collection of maternal viral load data started in 1998. Data on delivery and neonatal characteristics were recorded, including mode of delivery, sex, birth weight, gestational age, and duration of membrane rupture. Enrollments in the HAART era were defined as those that occurred on or after 1 January 1997.

Laboratory tests, including HIV RNA PCR, serologic testing, and CD4 cell count measurements, were performed locally, and the types of assays used were recorded. All laboratories were based in tertiary care hospitals (in most cases, university hospitals). Maternal CD4 cell counts and HIV RNA levels nearest to the time of delivery were used in the analyses here. CD4 cell counts were categorized as $<200,200-499$, and $\geqslant 500$ cells $/ \mathrm{mm}^{3}$. HIV-1 RNA levels were determined with the following assays: Roche Amplicor Monitor, versions 1.0 and 1.5 (Roche Diagnostic Systems); NASBA and NucliSens (Organon Teknika); and the Quantiplex branched chain DNA assay (Chiron Diagnostics). Women were classified as having undetectable viral loads on the basis of the appropriate cutoff values for the assay used.

Children who tested positive for a virological or serological marker of infection and/or children aged $>18$ months with persistence of antibody were considered to be infected $[17,18]$. If a child tested negative for HIV antibody and no virus or antigen had ever been detected, they were classified as uninfected. Premature delivery was defined as delivery before 37 weeks of gestation; gestational age was confirmed by ultrasonography, with data reported to the nearest completed week. Elective Caesarean section delivery was defined as Caesarean section delivery before rupture of membranes and before onset of labor. Duration of rupture of membranes was reported in hours and minutes, and the value was 0 for all elective Caesarean section deliveries. Multiple births (20 pairs of twins and 1 set of triplets) were treated as separate mother-child pairs.

Univariable comparisons for categorized variables were tested with the $\chi^{2}$ test or $\chi^{2}$ test for trends. Univariable and multivariable logistic regression analyses were used to obtain ORs and 95\% CIs. The optimal model for multivariable analysis was identified using the Akaike information criterion. All probability values were 2-tailed. Data entry was performed using Access 2000 (Microsoft), and analyses were made using SAS statistical software, version 8.02 (SAS Institute).

\section{RESULTS}

A total of 4525 mother-child pairs were enrolled in the European Collaborative Study up to May 2004, and 1983 (44\%) were enrolled during the HAART era (January 1997 to present). Most HIV-infected pregnant women who enrolled in the study in this latter period had acquired the virus via heterosexual transmission, knew they were infected when they became pregnant, and were moderately immunocompetent (table 1).

Figure 1 shows trends in HAART use and elective Caesarean section delivery rates during the HAART era. Although the rate of elective Caesarean section delivery increased from 1997 to 2000, patterns in the mode of delivery have been in a state of flux recently, with a decrease in the rate of elective Caesarean section delivery for the first time in $>1$ decade, although the rates started increasing again in 2003. Of the 1147 women overall who received HAART during pregnancy, 654 (57\%) started receiving HAART for the first time during pregnancy, $50(4 \%)$ had been receiving monotherapy or dual-drug therapy before their pregnancies, and the remaining 443 (39\%) were already receiving HAART when they became pregnant. The proportion of women who were receiving HAART before pregnancy increased from 5\% (11 of 217 women) in 1997, to $63 \%$ ( 213 of 338 women) in 2000, and to $85 \%$ (193 of 228 women) in 2003 ( $\chi^{2}$ test for trend, 436.8; $P<.001$ ).

Corresponding with increasing antenatal HAART use, there was a significant trend over time towards a higher prevalence of undetectable HIV RNA levels, from 29\% (30 of 102 women) in 1998 to $50 \%$ (99 of 199 women) in 2003 ( $\chi^{2}$ test for trend, 24.6; $P<.001$ ). In univariable analysis, detectability of maternal HIV RNA was associated with maternal use of antiretroviral therapy, CD4 cell count, and mode of delivery. Fourteen (19\%) of 73 women who had not received antenatal antiretroviral therapy had undetectable viral loads near the time of delivery, compared with 113 (30\%) of 383 of women receiving monotherapy or dual-drug therapy and 497 (51\%) of 966 women receiving HAART $\left(\chi^{2}\right.$ test, $72.7 ; P<.001$ ). A significantly higher proportion of women who were receiving HAART from before the pregnancy achieved undetectable viral loads, compared with 
Table 1. Maternal characteristics and delivery data for 1983 mother-child pairs enrolled in a study of mother-to-child transmission of HIV during the HAART era.

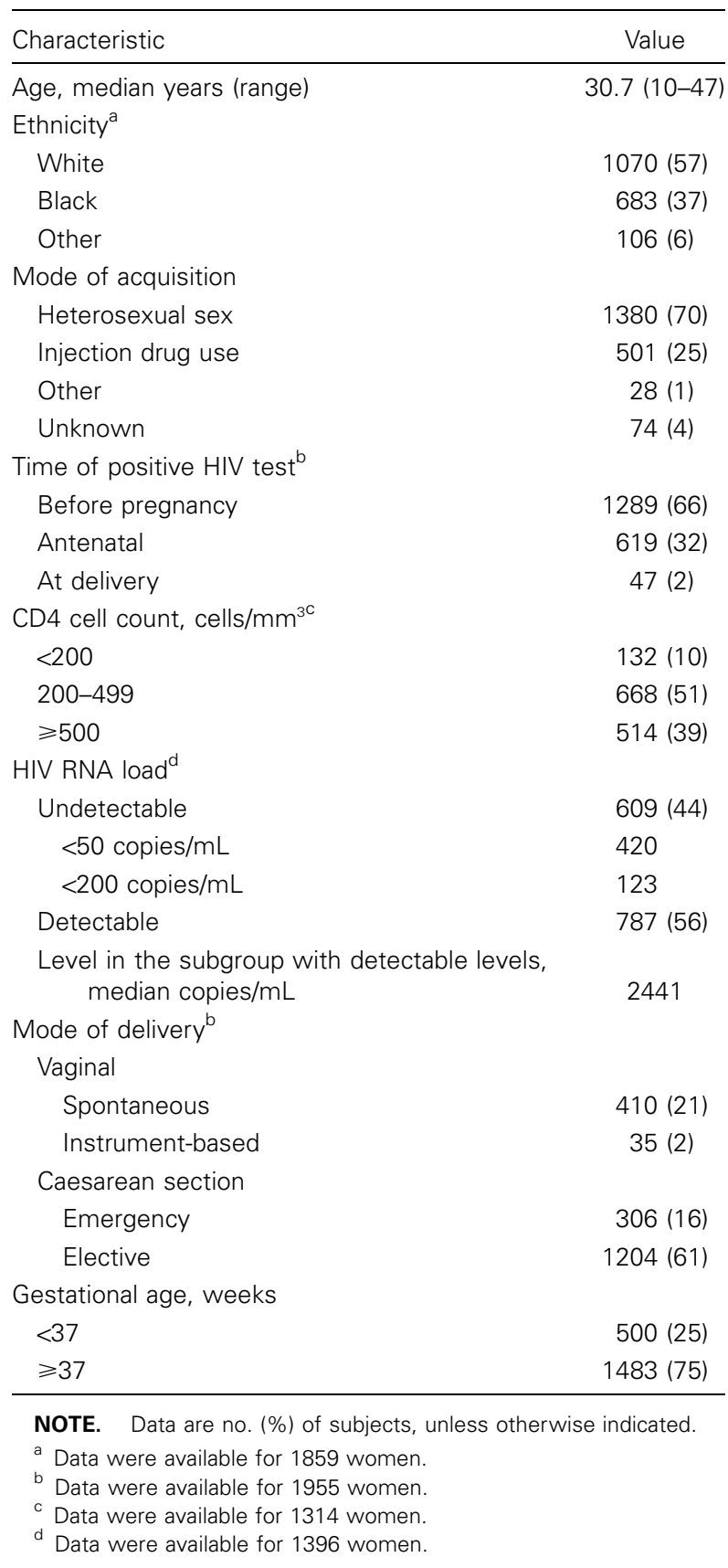

women who initiated HAART antenatally (234 [55\%] of 423 vs. $263[48 \%]$ of $543 ; \chi^{2}$ test, $\left.4.51 ; P=.03\right)$. Women delivering vaginally in the HAART era were more likely to have undetectable viral loads than were women who delivered by elective Caesarean section (142 [52\%] of 273 vs. 391 [43\%] of $918 ; \chi^{2}$ test, $7.55 ; P=.006)$.

Overall mother-to-child transmission rate and risks. The overall mother-to-child transmission rate was $2.87 \%(95 \%$ CI, $2.11 \%-3.81 \%$; 46 of 1602 cases), decreasing from $5.06 \%$
(95\% CI, $3.02 \%-7.87 \%$ ) in $1997-1998$ to $0.99 \%$ (95\% CI, $0.32 \%-2.30 \%)$ in 2001-2002. In univariable analysis, use of maternal antiretroviral therapy was significantly associated with a reduced mother-to-child transmission risk, with transmission of virus occurring for 18 (11.5\%) of 157 untreated mothers, compared with $11(1.20 \%)$ of 918 women who were receiving HAART ( $\chi^{2}$ test, 53.8; $P<.001$ ). Among the latter group, the risk of mother-to-child transmission was lower among those who initiated HAART before pregnancy than among women who initiated HAART during pregnancy (1 [0.25\%] of 397 vs. 10 [1.92\%] of $521 ; \chi^{2}$ test, $5.29 ; P=.02$ ). Mode of delivery was associated with vertical transmission, with infection occurring in $24(6.50 \%)$ of 369 infants delivered vaginally, 6 (2.51\%) of 239 delivered by emergency Caesarean section, and $16(1.65 \%)$ of 971 delivered by elective Caesarean section $\left(\chi^{2}\right.$ test, $22.4 ; P<.001)$.

Among the 369 infants delivered vaginally, mother-to-child transmission was not significantly associated with episiotomy (10 [7.5\%] of 123 infants with episiotomy infected, compared with 9 [4.8\%] of 179 infants without episiotomy; $\chi^{2}$ test, 1.04; $P=.31$ ) or instrument-based vaginal delivery (i.e., forceps or vacuum extraction, with 3 [10.3\%] of 29 infants with this type of delivery infected and 20 [5.9\%] of 318 infants with spontaneous delivery infected; $\chi^{2}$ test, $1.43 ; P=.70$ ). Details on the duration of rupture of membranes were available for 204 vaginally delivered infants. The median duration of rupture of membranes was $4.5 \mathrm{~h}$ for infected infants — significantly higher than the median duration for uninfected infants $(1.5 \mathrm{~h} ; P=$ .009 , by log rank test). Among the 207 infants who were delivered via emergency Caesarean section for whom there was information on duration of rupture of membranes, 50 were born with intact membranes with no transmissions, whereas 7 (4.27\%) of the remaining 157 infants (median duration of rupture of membranes, $5 \mathrm{~h}$ ) were infected. It is of interest to note that, among the 72 premature deliveries that involved women who were receiving HAART with vaginal or emergency Caesarean section deliveries and data on duration of rupture of membranes, 46 took place after rupture of membranes, with 1 infection $(2.17 \%)$, whereas there were no infections among the 26 premature infants born with intact membranes.

To investigate changes in the relative importance of traditional risk factors for mother-to-child transmission of HIV in the HAART era, multivariable logistic regression analyses were performed first for all 1880 mother-child pairs with information on maternal CD4 cell count, mode of delivery, maternal antiretroviral therapy, and prematurity and second for 885 mother-child pairs in the HAART era subgroup with viral load data available (table 2). Maternal viral load was the preeminent independent risk factor identified in the latter period, with a viral load of $>1000$ copies/mL associated with a 12 -fold increased risk of transmission. Elective Caesarean section delivery 


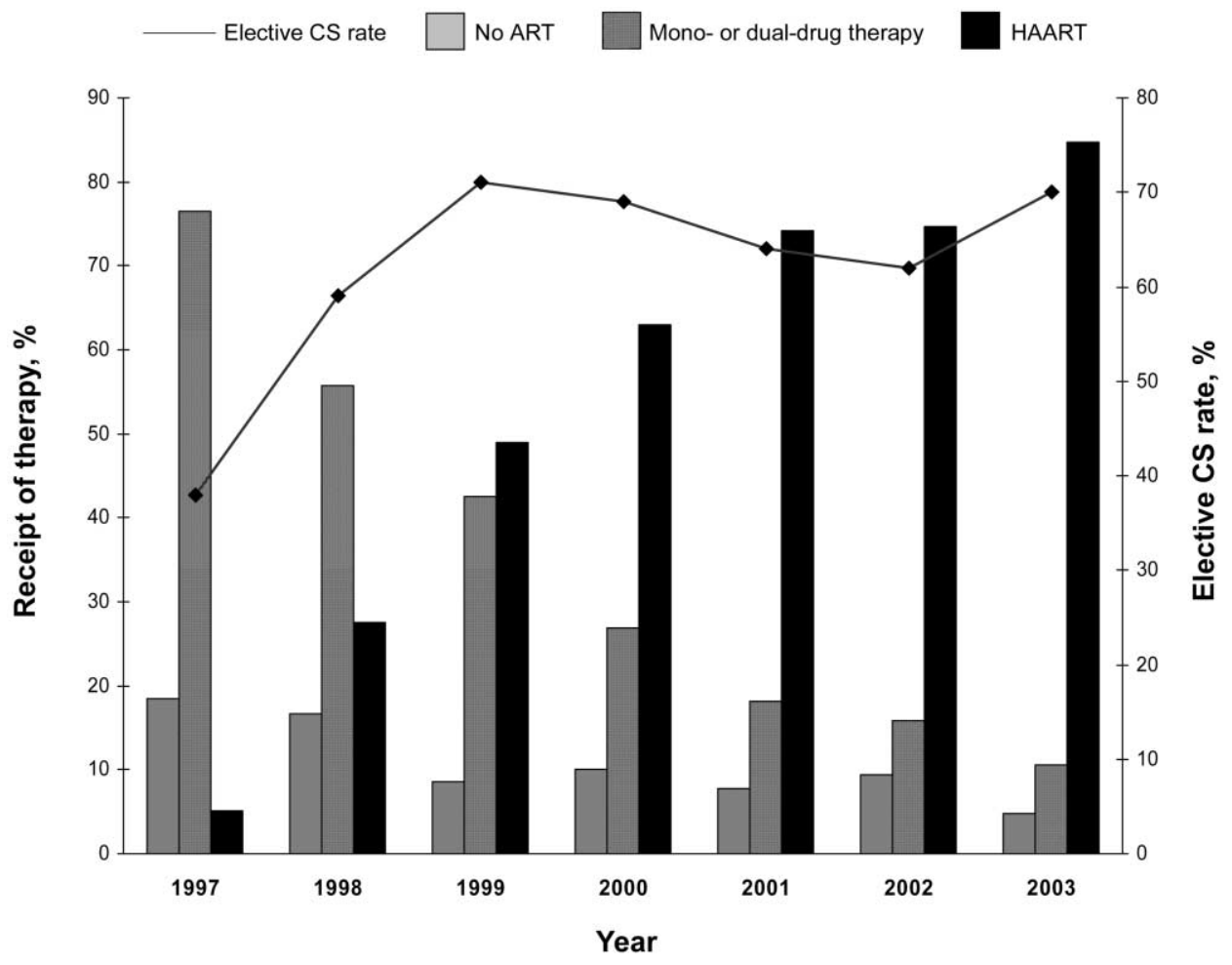

Figure 1. Trends in use of antenatal antiretroviral therapy (ART) and rates of elective Caesarean section (CS) deliveries, 1997-2003

was associated with a significant reduction (by two-thirds) in the risk of transmission, compared with vaginal delivery, independent of other variables. With the inclusion of maternal viral load, although there was a trend towards increased mother-to-child transmission risk associated with severe maternal immunosuppression, this did not reach statistical significance. Similarly, the pronounced reduction in the OR for transmission in the HAART subgroup did not achieve statistical significance, indicating that these variables work through maternal viral load. Reperformance of the subcohort analysis without the inclusion of maternal viral load resulted in adjusted ORs of a magnitude similar to that seen for the whole cohort, with significant reductions associated with elective Caesarean section delivery and HAART use. Prematurity was a significant independent risk factor in the whole cohort, and the direction of the effect was the same in the HAART era subgroup, although this finding was not statistically significant.

Elective Caesarean section delivery in women with undetectable HIV RNA levels and/or receipt of HAART. Among 560 women with undetectable HIV RNA levels (44\% with levels of $<50$ copies $/ \mathrm{mL}$ ), elective Caesarean section delivery was associated with a significantly reduced rate of mother-to-child transmission of HIV, compared with vaginal or emergency Caesarean section delivery, in univariable logistic regression analysis, with a $93 \%$ reduction in the risk of mother-to-child transmission (OR, 0.07; 95\% CI, 0.02-0.31; $P=.0004$ ). The small number of infected children $(n=27)$ in this subgroup precluded the inclusion of $>2$ categories of maternal antiretroviral therapy in multivariable analysis; however, with inclusion of a binary antiretroviral therapy variable (none vs. any), elective Caesarean section delivery was still associated with a halving of the mother-to-child transmission risk (adjusted OR, 0.52; 95\% CI, 0.14-2.03; $P=.358$ ). In a subanalysis limited to 759 mother-child pairs receiving antenatal HAART, elective Caesarean section delivery was associated with a $\sim 40 \%$ reduction in risk of mother-to-child transmission of HIV, compared with vaginal delivery (OR, 0.64; 95\% CI, 0.08-5.37; $P=.7$ ).

Children infected despite exposure to maternal HAART. Eleven children ( 6 male and 4 female children) became infected, even though their mothers received HAART antenatally; however, median duration of antenatal HAART was only 38 days (range, 4-152 days) for the 10 women who started receiving HAART during pregnancy, whereas 1 woman had been receiving therapy for $>1$ year before she became pregnant. The 11 women had relatively advanced HIV disease, with a median maternal CD 4 cell count of 209 cells $/ \mathrm{mm}^{2}$ (range, 64-468 cells/ $\mathrm{mm}^{3}$ ). Although elective Caesarean section delivery would have been the best mode of delivery for these infants in terms of prevention of mother-to-child transmission of HIV, only 5 infants were delivered by elective Caesarean section, with 4 infants delivered prematurely by emergency Caesarean section delivery and 1 delivered via spontaneous vaginal delivery at 38 weeks. 
Table 2. Logistic regression analyses of risk factors for mother-to-child transmission of HIV for the whole cohort and for the cohort of subjects enrolled during the HAART era alone.

\begin{tabular}{|c|c|c|c|c|c|c|}
\hline \multirow[b]{2}{*}{ Factor } & \multicolumn{3}{|c|}{$\begin{array}{l}\text { Whole cohort } \\
(n=1880)\end{array}$} & \multicolumn{3}{|c|}{$\begin{array}{l}\text { HAART era subcohort } \\
\qquad(n=885)\end{array}$} \\
\hline & $\begin{array}{l}\text { Unadjusted OR } \\
(95 \% \mathrm{Cl})\end{array}$ & $\begin{array}{l}\text { Adjusted OR } \\
(95 \% \mathrm{Cl})^{\mathrm{a}}\end{array}$ & $P$ & $\begin{array}{l}\text { Unadjusted OR } \\
(95 \% \mathrm{Cl})\end{array}$ & $\begin{array}{l}\text { Adjusted OR } \\
(95 \% \mathrm{CI})^{\mathrm{b}}\end{array}$ & $P$ \\
\hline \multicolumn{7}{|l|}{ Viral load, copies/mL } \\
\hline$<1000$ & $\ldots$ & $\ldots$ & & 1.00 & 1.00 & \\
\hline 1000-9999 & $\ldots$ & $\ldots$ & & $13.9(2.98-65.1)$ & $12.1(2.51-58.6)$ & .002 \\
\hline$\geqslant 10,000$ & $\ldots$ & $\ldots$ & & $15.5(3.27-74.0)$ & $12.1(2.31-63.1)$ & .003 \\
\hline \multicolumn{7}{|l|}{ Maternal CD4 cell count, cells $/ \mathrm{mm}^{3}$} \\
\hline$>500$ & 1.00 & 1.00 & & 1.00 & 1.00 & \\
\hline $200-499$ & $1.32(0.91-1.92)$ & $1.45(0.98-2.12)$ & .06 & $1.52(0.51-4.58)$ & $1.31(0.42-4.11)$ & .64 \\
\hline$<200$ & $2.04(1.27-3.28)$ & $2.01(1.23-3.29)$ & .003 & $4.00(1.13-14.1)$ & $2.68(0.70-10.2)$ & .14 \\
\hline \multicolumn{7}{|l|}{ Mode of delivery } \\
\hline Vaginal delivery & 1.00 & 1.00 & & 1.00 & 1.00 & \\
\hline Emergency Caesarean section & $0.61(0.37-1.00)$ & $0.73(0.42-1.24)$ & .24 & $0.56(0.14-2.20)$ & $0.51(0.12-2.18)$ & .37 \\
\hline Elective Caesarean section & $0.22(0.14-0.35)$ & $0.38(0.24-0.61)$ & $<.001$ & $0.40(0.15-1.08)$ & $0.33(0.11-0.94)$ & .040 \\
\hline \multicolumn{7}{|l|}{ Maternal antiretroviral therapy } \\
\hline None & 1.00 & 1.00 & & 1.00 & 1.00 & \\
\hline Monotherapy or dual-drug therapy & $0.36(0.23-0.56)$ & $0.49(0.31-0.76)$ & .002 & $0.43(0.11-1.68)$ & $0.56(0.13-2.39)$ & .44 \\
\hline HAART & $0.10(0.05-0.20)$ & $0.13(0.06-0.27)$ & $<.001$ & $0.17(0.04-0.65)$ & $0.37(0.09-1.56)$ & .18 \\
\hline \multicolumn{7}{|l|}{ Gestational age, weeks } \\
\hline$\geqslant 37$ & 1.00 & 1.00 & & 1.00 & 1.00 & \\
\hline$<37$ & $1.27(0.85-1.90)$ & $1.83(1.18-2.85)$ & .007 & $1.15(0.41-3.24)$ & $1.11(0.37-3.33)$ & .85 \\
\hline
\end{tabular}

a Adjusted for antenatal antiretroviral therapy, mode of delivery, prematurity, and maternal CD4 cell count.

b Adjusted for antenatal antiretroviral therapy, mode of delivery, prematurity, and maternal CD4 cell count and viral load.

All 11 neonates received antiretroviral prophylaxis (1 received zidovudine, lamivudine, and nevirapine for 6 weeks; 1 received zidovudine and lamivudine for 6 weeks; and the rest received oral zidovudine monotherapy [ 1 received it for 2 weeks, 1 received it for 4 weeks, and the remainder received it for 6 weeks]). Two mothers already had a child enrolled in the European Collaborative Study, and 1 of those children was also vertically infected.

\section{DISCUSSION}

In this large European cohort, there has been a dramatic decrease in the rate of mother-to-child transmission of HIV over time, to $\sim 1 \%$ in recent years, corresponding with the increasing uptake of prophylactic interventions. Nearly one-half of the women who enrolled in this study were already receiving HAART at the time that they became pregnant, and achievement of undetectable HIV RNA levels at or close to delivery was more frequent among women who started HAART before pregnancy than among women who started therapy during pregnancy. A high maternal viral load was the key risk factor for mother-to-child transmission of HIV, and the only other factor achieving statistical significance in the multivariable analysis in the HAART era was elective Caesarean section de- livery. In an analysis that allowed for maternal viral load, although HAART use was associated with a reduction of twothirds in the risk of mother-to-child transmission, this was not statistically significant, indicating that the effect of HAART is exerted through reducing the maternal plasma viral load.

Given the very small number of infections, due to widespread use of prophylactic interventions, it was not possible to confirm the statistical significance of effects such as prematurity in the HAART era. Although there is evidence to suggest that premature infants are more susceptible to vertical acquisition of HIV infection than are term infants, including the data presented here for the whole cohort $[15,16,19]$, the increased risk of mother-to-child transmission of HIV associated with prematurity in the HAART era here was not statistically significant. Our rate of premature birth was $25 \%$, reflecting the association previously reported between HAART use and duration of pregnancy [14]. Although there was a higher rate of mother-to-child transmission among infants delivered with episiotomy and/or forceps or vacuum, this did not reach statistical significance. However, this is likely to be associated with our sample size limitations, and we cannot exclude the possibility of a real risk associated with these types of delivery, as reported elsewhere in the pre-HAART era [20]. Our finding of an in- 
creased duration of rupture of membranes in infected infants, compared with uninfected infants, is consistent with previous reports [21].

The fluctuations over time in the mode of delivery seen in this cohort were caused by various factors. The temporary increase in the rate of emergency Caesarean section deliveries in 2000 was largely associated with premature labor or rupture of membranes of women booked for elective Caesarean section deliveries [14], which in turn resulted in a policy shift towards earlier scheduling of Caesarean section $[22,23]$. However, the recent increase in the number of vaginal deliveries seen and the finding that women who underwent vaginal deliveries were more likely to have undetectable viral loads than were those who underwent elective Caesarean section deliveries reflect the current lack of consensus regarding the additional benefit of elective Caesarean section delivery for women with undetectable viral loads who are receiving HAART [11-13]. Elective Caesarean section deliveries are associated with a higher rate of postpartum complications among HIV-infected women, compared with vaginal delivery [24, 25], although HIV-infected women are generally at an increased risk of postpartum complications, compared with uninfected women, regardless of mode of delivery [26]. In a recent study, the most common postpartum complication among infected women who underwent elective Caesarean section deliveries was anemia, which was strongly associated with receipt of antenatal antiretroviral therapy [26].

It is of interest to note that, even in the HAART era, we were able to show a significant reduction (two-thirds) in the risk of mother-to-child transmission of HIV associated with elective Caesarean section delivery, independent of HIV RNA load and maternal antiretroviral therapy use. In a meta-analysis involving women with plasma HIV RNA loads of $<1000$ copies/ $\mathrm{mL}$ at the time of delivery, Caesarean section delivery (with elective and emergency procedures grouped together) remained protective against mother-to-child transmission of HIV (with a $70 \%$ reduction in risk) after adjustment for maternal receipt of antiretroviral therapy (mainly non-HAART) and CD4 cell count [27]. In our cohort, the subanalyses involving women with undetectable viral loads close to delivery showed an effect of elective Caesarean section delivery in significantly reducing risk of transmission by $93 \%$, although small numbers and the resulting lack of power precluded additional adjustment for HAART use. To show a significant effect of elective Caesarean section delivery on mother-to-child transmission of HIV among women receiving HAART, an estimated 6345 and 7217 mother-child pairs would be needed with vaginal deliveries and elective Caesarean section deliveries, respectively, with $90 \%$ power [28], thus precluding any single study from answering the question of what the added benefit of elective
Caesarean section delivery might be for women receiving HAART.

Compartmentalization of HIV in the genital tract may explain the continuing effectiveness of elective Caesarean section delivery, even among women with undetectable plasma viral loads. Although a high correlation between plasma and genital tract viral loads has been reported in nonpregnant women [29], there is accumulating evidence to suggest there is a separate reservoir of the virus in the genital tract independent from plasma virus [30-32]. Understanding of the correlation between plasma viral load and the genital tract viral load in pregnancy [33] and of the differential effect of antiretroviral therapy is relatively limited, although several studies have suggested an association between genital tract viral load and mother-to-child transmission independent of plasma viral load [34, 35].

Despite initial optimism regarding the potential for "elimination" of vertically acquired HIV infection in resource-rich settings [36], we show that a very small number of infected women continue to transmit HIV to their infants. Among the 11 infants who were infected, despite exposure to maternal HAART and neonatal prophylaxis, there was a median exposure to antenatal antiretroviral therapy of slightly more than 1 month. Despite guidelines for universal antenatal screening for HIV infection [22], late identification of pregnant, HIV-infected women is still a barrier to optimum application of interventions in developed country settings $[37,38]$, in addition to lack of antenatal care in some subgroups of women [39].

The current strategy of offering an elective Caesarean section delivery to HIV-infected women in Europe [22] appears to be appropriate. In our cohort, approximately one-half of the women enrolled recently had detectable viral loads close to the time of delivery, and the continuing high rate of elective Caesarean section delivery indicates that these women are being recommended to undergo an elective Caesarean section delivery to prevent mother-to-child transmission of HIV. Although, in the presence of HAART, the absolute rate of mother-to-child transmission is likely to be low (in the region of $1 \%-2 \%$ ), our results suggest that elective Caesarean section delivery would further reduce this risk to $\sim 0.5 \%-1 \%$. However, with these very low overall rates, a large number of elective Caesarean section deliveries would need to be performed to prevent a single vertical infection. Although elective Caesarean section delivery has been shown to be cost-effective in women receiving zidovudine monotherapy and combination therapy, such analyses need to be repeated for women receiving HAART, because previous cost-benefit analyses have indicated that results are sensitive to variations in mother-to-child HIV transmission rates [40]. On an individual level, the decision regarding mode of delivery rests with the woman and her clinician, who should inform her of the potential risks and benefits. With adequate 
obstetric management, individual women may consider the benefit of a lower risk of infection in their infant to outweigh the potential disadvantages of an elective caesarean section.

\section{COLLABORATORS IN THE EUROPEAN COLLABORATIVE STUDY}

Dr. C. Giaquinto, Dr. E. Ruga, and A. De Rossi (Universita degli Studi di Padova, Italy); Prof. I. Grosch-Wörner (Charite Virchow-Klinikum, Berlin, Germany); Dr. J. Mok (Royal Hospital for Sick Children, Edinburgh, Scotland); Dr. I. de José, Dr. I. Bates, Dr. F. Hawkins, Dr. C. Ladrón de Guevara, Dr. J. Ma Peña, Dr. J. Gonzalez Garcia, Dr. J. R. Arribas Lopez, and Dr. M. C. Garcia-Rodriguez (Hospital Infantil La Paz, Madrid); Prof. F. Asensi-Botet, Dr. M. C. Otero, Dr. D. Pérez-Tamarit, and Dr. G. Suarez (Hospital La Fe, Valencia, Spain); Dr. H. Scherpbier, M. Kreyenbroek, and Dr. K. Boer (Academisch Medisch Centrum, Amsterdam, The Netherlands); Dr. A. B. Bohlin, Dr. S. Lindgren, Dr. A. Ehrnst, Dr. E. Belfrage, Dr. L. Navér, Dr. K. Lidman, and Dr. B. Anzen (Karolinska University Hospital, Huddinge and Solna, Sweden); Prof. J. Levy, Dr. P. Barlow, Dr. M. Hainaut, Dr. A. Peltier, and Dr. T. Goetghebuer (Hospital St. Pierre, Brussels, Belgium); Dr. A. Ferrazin and Prof. D. Bassetti (Department of Infectious Diseases, University of Genoa, Italy); Dr. A. De Maria (Department of Internal Medicine, University of Genoa, Italy); Dr. C. Gotta (Department of Obstetrics and Gynecology-Neonatology Unit, University of Genoa, Italy); Dr. A. Mûr, Dr. A. Payà, Dr. M. Viñolas, Dr. M. A. López-Vilchez, Dr. Rovira, and Dr. R. Carreras (Hospital del Mar, Universidad Autonoma, Barcelona, Spain); Dr. N. H. Valerius (Hvidovre Hospital, Denmark); Dr. J. Jimenez (Hospital 12 De Octubre, Madrid, Spain); Dr. O. Coll, Dr. A. Suy, Dr. J. M. Perez (Hospital Clinic, Barcelona, Spain); Dr. C. Fortuny (Hospital Sant Joan de Deu, Barcelona, Spain); Dr. M. Casellas Caro (Hospital Vall D’Hebron, Barcelona, Spain); Dr. Y. Canet (Hospital Parc Tauli de Sabadell, Barcelona, Spain); Dr. V. Savasi, Dr. A. Viganò, and Prof. E. Ferrazi (Ospedale L. Sacco, Milan, Italy); Dr. S. Alberico, Dr. M. Rabusin, and Dr. M. Bernardon (IRCCS Burlo Garofolo, Trieste, Italy); Dr. G. P. Taylor, Dr. E. G. H. Lyall (St Mary's Hospital, London); Ms. Z. Penn (Chelsea and Westminster Hospital, London); Drssa W. Buffolano and Dr. R. Tiseo (Pediatric Dept, Federico II University, Naples); Prof. P. Martinelli, Dr. A. Agangi, and Drssa. M. Sansone (Obstetric Dept, Federico II University, Naples, Italy); Dr. C. Tibaldi, Dr. S. Marini, Dr. G. Masuelli, and Dr. C. Benedetto (University di Torino, Italy); Dr. T. Niemieç (National Research Institute of Mother \& Child, Warsaw, Poland); and Dr. M. Marczynska and Dr. A. Horban (Medical University of Warsaw, Poland).

\section{Acknowledgments}

We thank Prof. L. Chieco-Bianchi, Prof. F. Zacchello, Dr. E. Ruga, Dr. R. D’Elia, Dr. A. M. Laverda, Dr. A. Mazza, and Mrs. S. Oletto (Padua, Italy); Dr. Cornelia Feiterna and Dr. Ralf Weigel (Berlin, Germany); Dr. S. Burns, Dr. N. Hallam, Dr. P. L. Yap, and Dr. J. Whitelaw (Edinburgh, Scotland); Dr. B. Sancho and Dr. G. Fontan-Casanego (Madrid, Spain); Dr. A. Gonzalez Molina, Dr. M. Gobernado, Dr. J. L. Lopez, and Dr. J. Cordoba (Valencia, Spain); A. van der Plas (Amsterdam, The Netherlands); Dr. K. Elfgren, Prof. U. Ewald, Dr. A. C. Lindholm, Dr. P. O. Pehrson, and Dr. K. Gyllensten (Sweden); Dr. G. Di Siena, Dr. E. Pontali, Prof. M. F. Pantarotto, G. Mantero, and Dr. P. Dignetti (Genoa, Italy); Dr. M. Guxens, Dr. P. Martinez, Dr. S. Pisa, Dr. B. Martinez de Tejada, Dr. L. Zamora, and Dr. R. Vidal (Barcelona, Spain); Dr. G. Zucotti (Milan, Italy); Prof. P. A. Tovo and Dr. C. Gabiano (Turino, Italy); Dr. E. Pagliaro and Dr. M. T. Melisi (Naples, Italy); and The Regional Health Office and RePuNaRC (Naples).

Financial support. The European Collaborative Study is a concerted action of the European Commission (QLK2-CT-2000-00002). The Medical Research Council (UK) Sexual Health and HIV Research Strategy Committee provided support to the European Collaborative Study coordinating center.

Potential conflicts of interest. All authors: no conflicts.

\section{References}

1. Dorenbaum A, Cunningham CK, Gelber RD, et al. Two-dose intrapartum/newborn nevirapine and standard antiretroviral therapy to reduce perinatal HIV transmission: a randomized trial. JAMA 2002; 288: 189-98.

2. Simpson BJ, Shapiro DE. Prospective cohort study of children born to HIV-infected mothers, 1985 through 1997: trends in the risk of vertical transmission, mortality and acquired immunodeficiency syndrome indicator diseases in the era before highly active antiretroviral therapy. Pediatr Infect Dis J 2000; 19:618-24.

3. Mandelbrot L, Landreau A, Rekacewicz C, et al. Lamivudine-zidovudine combination for prevention of maternal-infant transmission of HIV-1. JAMA 2001; 285:2083-93.

4. Cooper ER, Charurat M, Mofenson LM, et al. Combination antiretroviral strategies for the treatment of pregnant HIV-1-infected women and prevention of perinatal HIV-1 transmission. J Acquir Immune Defic Syndr 2002; 29:484-94.

5. Centers For Disease Control and Prevention. HIV/AIDS surveillance report, 2002. Atlanta, GA: Centers For Disease Control and Prevention, 2003.

6. European Centre for the Epidemiological Monitoring of AIDS. HIV/ AIDS Surveillance in Europe: midyear report 2003. Paris: World Health Organization and United Nations AIDS Collaborating Centre, 2003.

7. Watts DH, Balasubramanian R, Maupin RT, et al. Maternal toxicity and pregnancy complications in HIV-infected women receiving antiretroviral therapy: an analysis of the PACTG 316 Study. Am J Obstet Gynecol 2004; 190:506-16.

8. Mode of delivery and vertical transmission of HIV-1: a meta-analysis from fifteen prospective cohort studies. The International Perinatal HIV Group. Lancet 1999; 340:977-87.

9. The European Mode of Delivery Collaboration. Elective caesarean section versus vaginal delivery in preventing vertical HIV-1 transmission: a randomised clinical trial. Lancet 1999;353:1035-9.

10. Dominguez KL, Lindegren ML, D'Almada PJ, et al. Increasing trend of cesarean deliveries in HIV-infected women in the United States from 1994 to 2000. J Acquir Immune Defic Syndr 2003;33:232-8.

11. Rowland BL, Vermillion ST, Soper DE. Scheduled cesarean delivery and the prevention of human immunodeficiency virus transmission: a survey of practicing obstetricians. Am J Obstet Gynecol 2001; 185: $327-31$. 
12. Stringer JSA, Rouse DJ, Goldenberg RL. Prophylactic cesarean delivery for the prevention of perinatal human immunodeficiency virus transmission: the case for restraint. JAMA 1999;281:1946-9.

13. Landers DV, Duarte G. Mode of delivery and the risk of vertical transmission of HIV-1 [correspondence]. N Engl J Med 1999;341:205-6.

14. European Collaborative Study and the Swiss Mother and Child HIV Cohort Study. Combination antiretroviral therapy and duration of pregnancy. AIDS 2000; 14:2913-20.

15. Kuhn L, Steketee RW, Weedon J, et al. Distinct risk factors for intrauterine and intrapartum human immunodeficiency virus transmission and consequences for disease progression in infected children. J Infect Dis $1999 ; 179: 52-8$.

16. Goedert JJ, Mendez H, Drummond JE, et al. Mother-to-infant transmission of human immunodeficiency virus type 1: association with prematurity or low anti-gp120. Lancet 1989;2:1351-4.

17. European Collaborative Study. HIV-infected pregnant women and vertical transmission in Europe since 1986. AIDS 2001; 15:761-70.

18. European Collaborative Study. Are there gender and race differences in cellular immunity patterns over age in infected and uninfected children born to HIV-infected women? J Acquir Immune Defic Syndr 2003; 33:635-41.

19. European Collaborative Study. Maternal viral load and vertical transmission of HIV-1: an important factor but not the only one. AIDS 1999; 13:1377-85.

20. Mandelbrot L, Mayaux MJ, Bongain A, et al. Obstetric factors and mother-to-child transmission of human immunodeficiency virus type 1: the French perinatal cohorts. Am J Obstet Gynecol 1996; 175:661-7.

21. The International Perinatal HIV Group. Duration of ruptured membranes and vertical transmission of HIV-1: a meta-analysis from fifteen prospective cohort studies. AIDS 2001; 15:357-68.

22. Newell ML, Rogers MF. Pregnancy and HIV infection: a European consensus on management. AIDS 2002; 16:S1-18.

23. European Collaborative Study. Pregnancy-related changes in the longer-term management of HIV infected women in Europe. Eur J Obstet Gynecol Reprod Biol 2003; 111:3-8.

24. Marcollet A, Goffinet F, Firtion G, et al. Differences in postpartum morbidity in women who are infected with the human immunodeficiency virus after elective cesarean delivery, emergency cesarean delivery, or vaginal delivery. Am J Obstet Gynecol 2002; 186:784-9.

25. Read JS, Tuomala R, Kpamegan E, et al. Mode of delivery and postpartum morbidity among HIV-infected women: the women and infants transmission study. J Acquir Immune Defic Syndr 2001; 26:236-45.

26. European HIV in Obstetrics Group. Higher rates of post-partum complications in HIV infected than in uninfected women irrespective of mode of delivery. AIDS 2004; 18:933-8.
27. Ioannidis JPA, Abrams EJ, Bulterys M, et al. Perinatal transmission of human immunodeficiency virus type 1 by pregnant women with RNA virus loads $<1000$ copies/mL. J Infect Dis 2001; 183:539-45.

28. Fleiss JL, Tytun A, Ury HK. A simple approximation for calculating sample sizes for comparing independent proportions. Biometrics 1980;36:343-6.

29. Debiaggi M, Zara F, Spinillo A, et al. Viral excretion in cervicovaginal secretions of HIV-1-infected women receiving antiretroviral therapy. Eur J Clin Microbiol Infect Dis 2001;20:91-6.

30. Hart CE, Lennox JL, Pratt-Palmore M, et al. Correlation of human immunodeficiency virus type 1 RNA levels in blood and the female genital tract. J Infect Dis 1999; 179:871-2.

31. Kovacs AAS, Wasserman SS, Wright DJ, et al. Determinants of HIV1 shedding in the genital tract of women. Lancet 2001;358:1593-601.

32. Fiore J, Suligoi B, Saracino A, et al. Correlates of HIV-1 shedding in cervicovaginal secretions and effects of antiretroviral therapies. AIDS 2003; 17:2169-76.

33. Garcia-Bujalance S, Ruiz G, De Guevara CL, et al. Quantitation of human immunodeficiency virus type 1 RNA loads in cervicovaginal secretions in pregnant women and relationship between viral loads in the genital tract and blood. Eur J Clin Microbiol Infect Dis 2004; 23: $111-5$.

34. Tuomala RE, O'Driscoll PT, Bremer JW, et al. Cell-associated genital tract virus and vertical transmission of human immunodeficiency virus type 1 in antiretroviral-experienced women. J Infect Dis 2003; 187: 375-84.

35. Chuachoowong R, Shaffer N, Siriwasin W, et al. Short-course antenatal zidovudine reduces both cervicovaginal human immunodeficiency virus type 1 RNA levels and risk of perinatal transmission. J Infect Dis 2000; 181:99-106.

36. Mofenson LM. Can perinatal HIV infection be eliminated in the United States? JAMA 1999;282:577-9.

37. Peters VB, Liu KL, Dominguez KL, et al. Missed opportunities for perinatal HIV prevention among HIV-exposed infants born 1996-2000, pediatric spectrum of HIV disease cohort. Pediatrics 2003;111: 1186-91.

38. Cliffe S, Tookey PA, Nicoll AG. Antenatal detection of HIV: national surveillance and unlinked anonymous survey. BMJ 2001;323:376-7.

39. Rajegowda BK, Das BB, Lala R, Rao S, McNeely DF. Expediated human immunodeficiency virus testing of mothers and newborns with unknown HIV status at the time of labour and delivery. J Perinat Med 2000; 28:458-63.

40. Halpern MT, Read JS, Ganoczy DA, Harris DR. Cost-effectiveness of cesarean section delivery to prevent mother-to-child transmission of HIV-1. AIDS 2000; 14:691-700. 